\title{
Implementação do Pró-Saúde no Curso de Odontologia da Universidade Estadual de Maringá
}

Raquel Sano Suga Terada*, Mitsue Fujimaki Hayacibara**, Cynthia Junqueira Rigolon***, Mariliani Chicarelli da Silva****, Luiz Fernando Lolli*****, Mirian Marubayashi Hidalgo $* * * * * *$

* Doutora em Odontologia (Dentística) pela Universidade de São Paulo (FOB/USP) e Professora Associada do Departamento de Odontologia da Universidade Estadual de Maringá

** Doutora em Cariologia pela Faculdade de Odontologia de Piracicaba (UNICAMP) e Professora Adjunta da área de Saúde Coletiva do Departamento de Odontologia da Universidade Estadual de Maringá

*** Mestre em Odontologia pela Universidade de São Paulo (FOB/ USP-UEL), Professora Auxiliar da área de Saúde Coletiva do Departamento de Odontologia da Universidade Estadual de Maringá

**** Doutora em Radiologia Odontológica pela Faculdade de Odontologia de Piracicaba (UNICAMP) e Professora Adjunta do Departamento de Odontologia da Universidade Estadual de Maringá

***** Doutor em Odontologia Preventiva e Social pela Universidade Estadual Paulista (UNESP/Araçatuba), Professor Adjunto da área de Saúde Coletiva do Departamento de Odontologia da Universidade Estadual de Maringá e Professor Adjunto da área de Odontologia Legal da UNINGÁ (Faculdade Ingá)

******* Doutora em Odontologia (Patologia Bucal) pela Universidade de São Paulo (FOB/USP) e Professora Associada da área de Endodontia do Departamento de Odontologia da Universidade Estadual de Maringá

\section{RESUMO}

Este trabalho objetivou relatar a implementação do Pró-saúde no curso de Odontologia da Universidade Estadual de Maringá (UEM) e as atividades realizadas até o ano 2010. Trata-se de um estudo documental retrospectivo de consulta a relatórios técnicos e financeiros das duas cartas acordos do projeto. Uma equipe de seis docentes do Departamento de Odontologia da UEM se encarregou de avaliar todo o material e relacionar as principais ações desenvolvidas. Neste processo, foram fundamentais as constituições de comitês gestor e de acompanhamento e a contratação de assessorias administrativa e pedagógica de recursos humanos. Dentre as ações, destacam-se a atuação nas atividades extramurais, a re-inserção de acadêmicos nos serviços municipais de saúde de Maringá, o esta- belecimento de parceria com a Secretaria de Saúde de Marialva, a criação da Clínica Ampliada em Odontologia, os fóruns para relato de experiência e avaliação, os levantamentos epidemiológicos e o projeto de heterocontrole das águas de abastecimento público de Maringá. Conclui-se que a implantação do Pró-Saúde serviu como marco inicial do processo de mudança com a constituição de uma massa crítica de docentes engajados na formação dos profissionais de saúde e trabalhando de forma colegiada. Persistem como desafios a integração multiprofissional e a mudança do paradigma curativista para o de promoção de saúde.

\section{DESCRITORES}

Recursos humanos em saúde. Odontologia. Política de educação superior. 
$\mathbf{0}$ desafios na formação de profissionais de saúde para o século XXI incluem a necessidade de superar as grandes lacunas e desigualdades que persistem em todo mundo, onde uma grande parte dos 7 bilhões de habitantes ainda estão presos às condições de saúde do século passado. Neste contexto, a reforma educacional é um processo longo e difícil, porém, segundo o relatório da Comissão Independente sobre a Educação dos Profissionais de Saúde para o Século XXI, divulgado em novembro de 2010 na revista Lancet, ${ }^{1}$ a aprendizagem transformadora e a compreensão da interdependência são duas ideias básicas que fortalecerão os sistemas de saúde.

Discutir a formação dos profissionais de saúde é de co-responsabilidade dos setores saúde e de educação, há de se desejar uma retroalimentação nos moldes de parceria intersetorial, em que o ensino, minimamente se responsabilize com o desenvolvimento de um processo ensino-aprendizagem significativo para o aluno, criativo e comprometido com as necessidades locorregionais de saúde, e incentive a autonomia e auto-gestão do próprio aprender. ${ }^{2}$ Diante da necessidade de mudança no perfil dos egressos que passam pelas instituições de ensino, várias iniciativas sobre os rumos da educação na área da saúde têm sido experimentadas há pelo menos trinta anos, inicialmente na área médica e, atualmente, direcionadas a todas as demais áreas. Este movimento tem ocorrido internacionalmente, apoiado também pela Organização Mundial de Saúde. No Brasil, em 2005, a Portaria Interministerial ${ }^{\circ} 2.101$, de 03 de novembro instituiu o Programa Nacional de Reorientação da Formação Profissional em Saúde - Pró-Saúde, ${ }^{3}$ para os cursos de Medicina, Enfermagem e Odontologia, cursos vinculados à estratégia Saúde da Família, com objetivo de melhorar a formação de recursos humanos, adequando-os às necessidades da população brasileira, visando o fortalecimento do Sistema Único de Saúde (SUS).

Os Cursos de Medicina e Odontologia da Universidade Estadual de Maringá (UEM) tiveram seus projetos aprovados no edital de 2005, sendo que a implementação do programa na Odontologia vem acontecendo de forma gradativa e com a participação efetiva dos docentes, discentes, serviço e comunidade. No entanto, nenhum ensaio apresentando a trajetória, com seus avanços, ações e dificuldades, ainda foi publicado. Desta forma, o objetivo deste trabalho foi relatar a história da implantação do Pró-Saúde no Curso de Odontologia da UEM bem como as ações desenvolvidas até o momento.

\section{METOLODOGIA}

Trata-se de um estudo documental, retrospectivo, no qual foram consultados os relatórios técnicos e financeiros referentes à $1^{\mathrm{a}}$ e $2^{\mathrm{a}}$ Cartas Acordo do projeto Pró-Saúde Odontologia - UEM, encaminhados, respectivamente, ao Departamento de Gestão da Educação na Saúde do Ministério da Saúde e Organização Pan-americana de Saúde, nos anos de 2008 e 2010, e todos os documentos do Comitê Gestor e de Acompanhamento do referido projeto, incluindo ofícios, atas, memórias de reuniões e relatórios. A análise ocorreu por uma equipe de seis docentes do Departamento de Odontologia, diretamente vinculados às ações desenvolvidas desde a contemplação do PróSaúde até o ano de 2010.

Os avaliadores destacaram coletivamente as principais ações desenvolvidas bem como a aplicação dos recursos financeiros. Além disso, foram relacionadas as avaliações realizadas durante a implementação do projeto, as estratégias adotadas e as principais dificuldades ou nós-críticos para o avanço das iniciativas de mudança.

Os dados foram apresentados em formato textual, considerando a facilidade de exposição e contextualização de idéias, nas variáveis:

- breve histórico da implantação,

- principais ações desenvolvidas,

- resumo orçamentário e

- avaliação do projeto e impacto no curso.

\section{RESULTADOS \\ Breve histórico da implantação do projeto}

A proposta inicial foi elaborada por um pequeno grupo de docentes, alguns envolvidos com o movimento dos ativadores de mudança na formação profissional, outros na coordenação do curso, como também docentes da área de Saúde Coletiva, durante reuniões realizadas no ano de 2005 .

Nos dias 23 e 24 de novembro de 2006, o curso recebeu a primeira visita técnica do Ministério da Saúde e, na ocasião, as assessoras indicaram a necessidade e importância da implantação de um Comitê Gestor e de Acompanhamento do Pró-Saúde. Assim, a partir de 22 de janeiro de 2007, ocorreu a primeira reunião do Comitê de Acompanhamento do PróSaúde da UEM. Esta iniciativa foi fundamental, pois além de institucionalizar o projeto com a Pró-Reitoria de Ensino da UEM, criou-se um espaço para integração dos cursos que haviam recebido aprovação da proposta para o Pró-Saúde; Odontologia e Medicina. 
Posteriormente, o curso de Enfermagem foi convidado a participar deste Comitê, pois havia sido contemplado no edital do Pró-Saúde 2.

\section{Principais ações desenvolvidas}

$\mathrm{Na} 1^{\text {a }}$ Carta Acordo, todos os projetos previstos para o Eixo A - Orientação Teórica foram realizados. Um ponto de destaque foi a participação no "I Fórum Paranaense Pró-Saúde Odontologia”, em Londrina ${ }^{4}$. Outro marco importante foi o "II Fórum Parananense do Pró-Saúde da Odontologia", ${ }^{5}$ realizado em Maringá no ano de 2008, quando ocorreu a "Oficina das Diretrizes Curriculares Nacionais para os Cursos de Odontologia”, coordenada pelo Prof Léo Krieger e a Profa Maria Celeste Morita, seguindo os moldes das oficinas da ABENO. Outras atividades de extensão, como "Sistema Único de Saúde como Referência e a Formação Profissional para o SUS" e "A Política Nacional de Humanização e a Formação Profissional de Saúde" foram fundamentais para sensibilizarem principalmente a academia sobre os rumos da formação em saúde. Além disso, outros projetos, inicialmente não previstos, foram surgindo e sendo desenvolvidos.

No Eixo B - Cenários de Prática, destacaram-se algumas fortalezas:

- realização de atividades extramurais em escolas e na comunidade desde o segundo ano do curso;

- diálogo aberto e efetiva parceria com os municípios de Maringá e Marialva, onde ocorrem os estágios em unidades básicas de saúde com os estudantes do quarto e quinto ano;

- atuação de equipes de saúde bucal da Odontologia/UEM junto à equipe 21 da casa do PSF, da UBS Mandacaru em Maringá;

- participação de discentes e docentes nas reuniões do conselho municipal e local de saúde;

- desenvolvimento de mecanismos institucionais de referência e contra-referência com a rede do SUS.

No Eixo C - Orientação pedagógica, todos os projetos previstos também foram realizados. Uma ação fundamental, tanto para este eixo quanto para o Eixo A, foi a contratação de Assessoria Pedagógica para avaliação da implementação do Pró-Saúde e Assessoria Administrativa para gestão de recursos humanos.

Considerando a $2^{\text {a }}$ Carta Acordo, no Eixo A Orientação Teórica, destacaram-se os "levantamentos epidemiológicos das condições de saúde bucal da população de Maringá e Marialva" realizados em parceria com as coordenações de saúde bucal dos referidos municí- pios. Em ambos os locais, foram os primeiros dados epidemiológicos que seguiram a metodologia preconizada pela Organização Mundial de Saúde e os levantamentos do $\mathrm{SB}$ - Brasil. Igualmente, uma pesquisa relevante foi iniciada em Julho/2009 sobre o heterocontrole da fluoretação da água de abastecimento público em Maringá. Este trabalho extende-se até o momento. Ainda neste eixo, foram produzidos dois vídeos institucionais, um sobre o Conselho Local de Saúde e outro sobre o Projeto Lebu, um projeto de extensão que é referência de diagnóstico e tratamento na área de Estomatologia para todos os 30 municípios da $15^{\text {a }}$ Regional de Saúde do Paraná e alguns de outras regionais do Estado.

No Eixo B - Cenários de Prática, foi dada continuidade aos estágios supervisionados nas unidades de saúde. Como ação nova mais relevante neste momento, ocorreu a implantação da Clínica Ampliada do Curso de Odontologia, a primeira do Brasil. O principal objetivo desta é organizar o serviço de referência e contra-referência da Clínica Odontológica do curso para um cuidado integral, humanizado e resolutivo, tendo em vista os pressupostos da Clínica Ampliada da Política Nacional de Humanização do Sistema Único de Saúde.

No Eixo C-Orientação Pedagógica, destacaram-se três oficinas realizadas em conjunto com os projetos Pró-Saúde Medicina e Enfermagem:

- "II Oficina do Comitê de Acompanhamento do PróSaúde",

- "I Fórum de Orientação Pedagógica para Docentes da Área da Saúde" e a

- "Oficina Pró-Saúde, FNEPAS e a formação de recursos humanos para o Sistema Único de Saúde".

Durante a execução da $2^{\mathrm{a}}$ Carta Acordo o curso se inseriu, juntamente com os cursos de Medicina, Enfermagem, Farmácia, Psicologia e Educação Física, nos Programas de Educação pelo Trabalho para a Saúde (PET-Saúde) de Maringá e Marialva e, desde então, tem-se buscado esforços para um trabalho conjunto, fato que deverá se potencializar na $3^{\text {a }}$ Carta Acordo. Igualmente ao disposto na $1^{\text {a }}$ Carta Acordo, contou-se com apoio das assessorias pedagógica e administrativa de recursos humanos, fundamentais para êxito das ações.

\section{Resumo orçamentário}

No projeto elaborado em 2005, o montante total de recursos aprovado foi de $\mathrm{R} \$ 1.243 .879,00$, dividido em três anos, sendo $R \$ 399.730,00, \mathbf{R} \$ 425.350,00$ e 
$\mathrm{R} \$ 418.799,00$, respectivamente, para o primeiro, segundo e terceiro anos.

$\mathrm{Na} 1^{\text {a }}$ Carta Acordo, $100 \%$ dos recursos foram empreendidos. Já na $2^{\mathrm{a}}$ Carta Acordo, o pagamento foi dividido em três parcelas, respectivamente, de $\mathrm{R} \$ 202.252,50, \mathrm{R} \$ 180,562,50$ e $\mathbf{R} \$ 42.535,00$. Utilizouse somente $90 \%$ do recurso devido a dificuldade no recebimento da segunda parcela e o não recebimento da terceira, pois não houve tempo hábil para a tramitação dos documentos. O tempo para execução da $2^{\mathrm{a}}$ carta acordo foi de 8 meses.

$\mathrm{Na} 1^{\text {a }}$ Carta Acordo, $72 \%$ dos recursos foram destinados a materiais permanentes. Istojustifica-se, pois a intenção era estruturar os campos de estágio a receberem os estudantes, promovendo a reaproximação e fortalecimento dos laços da academia com o serviço municipal de saúde. Os eventos promovidos foram mais pontuais, com o objetivo principal de sensibilizar os atores envolvidos acerca da necessidade de mudança no perfil do egresso a ser formado. Neste período, a maior dificuldade foi lidar com a parte burocrática e gerencial dos recursos financeiros, pelo grande volume orçamentário e, principalmente, pela forma com que o convênio foi estabelecido, ou seja,

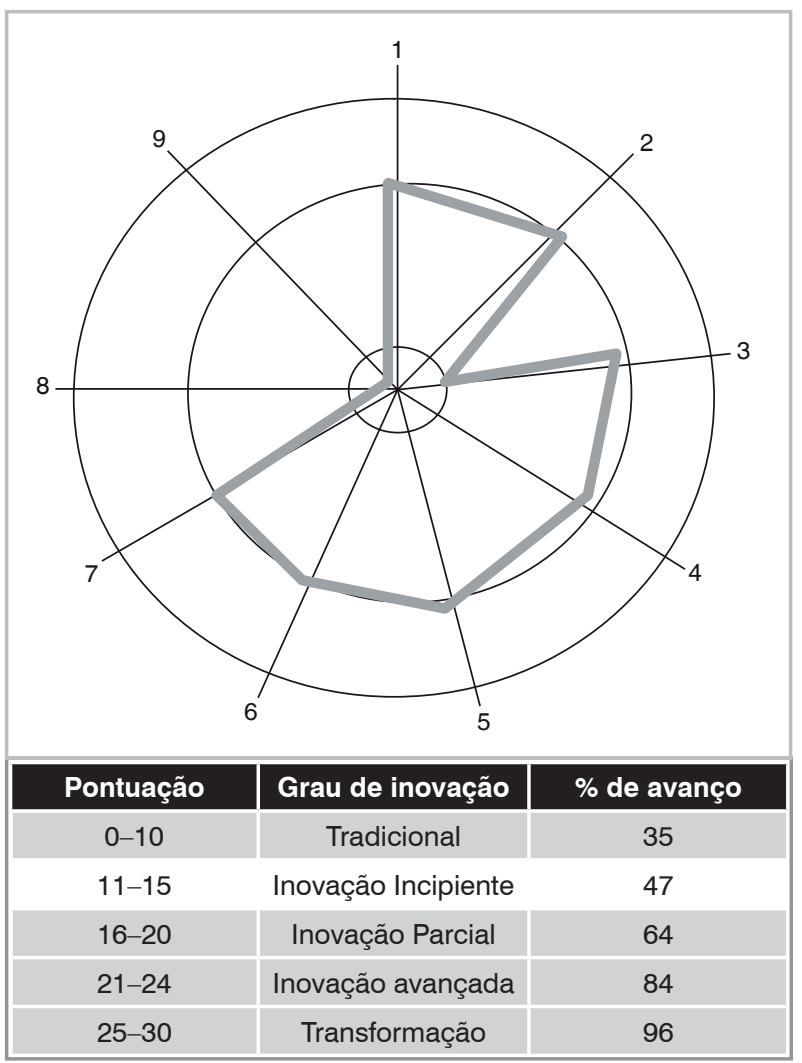

Figura 1 - Diagnóstico inicial do grau de inovação do Curso de Odontologia - Dezembro/2005. pela instituição e não por uma fundação. Por outro lado, mesmo com inexperiência dos principais envolvidos à frente do projeto sobre o funcionamento da máquina administrativa universitária, os gastos orçamentários foram executados com êxito.

$\mathrm{Na} 2^{\text {a }}$ Carta Acordo, $85 \%$ dos recursos foram destinados à custeio, totalizando $\mathrm{R} \$ 361.547,50 \mathrm{e}$, para material permanente, $15 \%$, o que representa $\mathrm{R} \$ 63.802,50$. Destacam-se os eventos realizados para a implementação da Clínica Ampliada do Curso de Odontologia da UEM.

\section{Avaliação do projeto e impacto no curso}

O diagnóstico Inicial do Curso de Odontologia UEM, definido pelo perfil radial, a fim de elaborar o projeto de participação no Pró-Saúde, apresentava-se, como demonstrado na Figura 1, com um grau de inovação incipiente.

Ao analisar os dados da aplicação do perfil radial, obtidos no evento I Seminário de Avaliação do Curso de Odontologia da UEM (outubro de 2007), percebeu-se um avanço na pontuação (16) o que permitiu verificar a inovação parcial que começava a ocorrer dentro do curso, conforme apresentado na Figura 2.

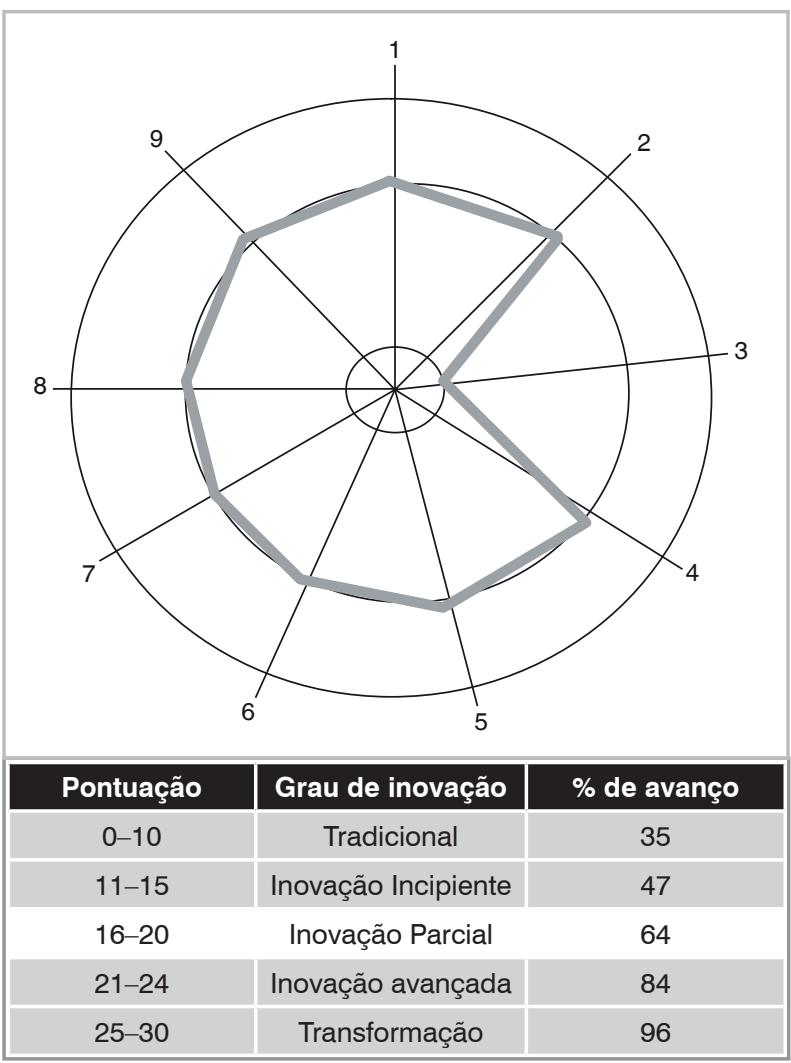

Figura 2 - Perfil radial do grau de inovação - Outubro/2007. 


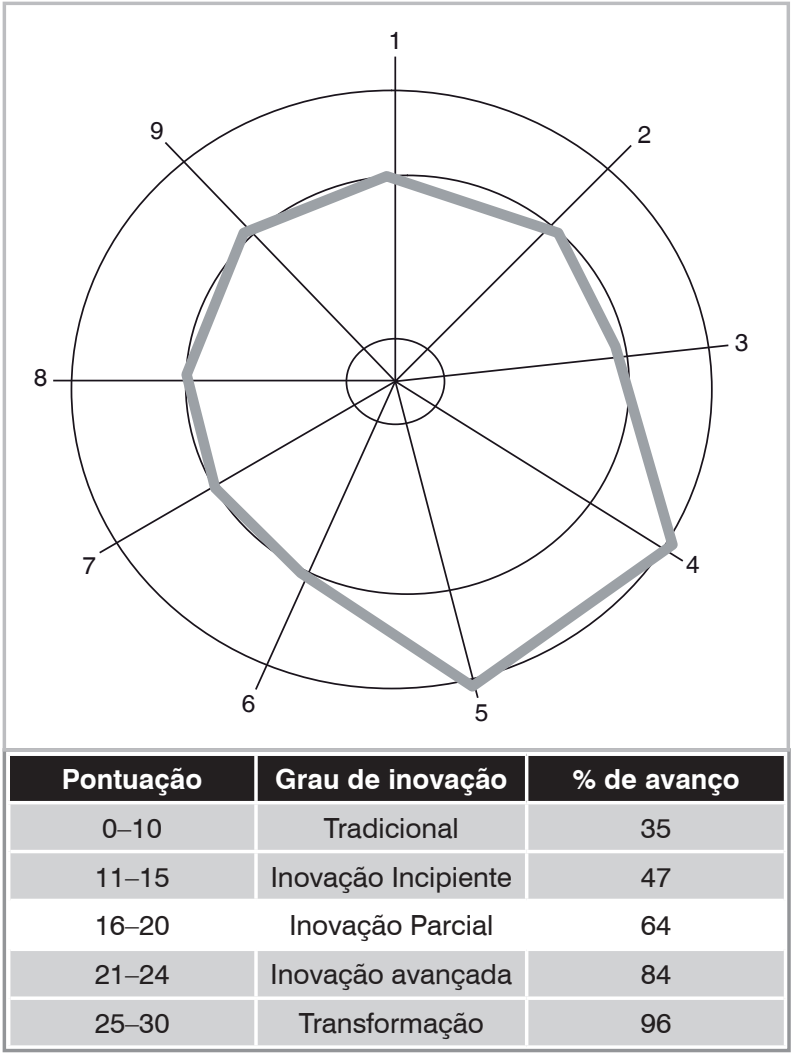

Figura 3 - Perfil radial do grau de inovação em Novembro/2008.

Em novembro de 2008, realizou-se uma terceira avaliação e a partir da análise das percepções e dados obtidos avançaram-se mais quatro pontos, em relação ao perfil anterior (Figura 3).

O comitê gestor decidiu dar continuidade à forma de avaliação já empregada nos anos anteriores, até que um instrumento de avaliação indicado por órgãos superiores seja sugerido. Nesse sentido, a partir da estrutura matricial de eixos, vetores e estágios de desenvolvimento, sugerida pelo Programa, em março de 2010 foram organizados encontros com alunos, professores e prestadores de serviços bem como, elaborados instrumentos de coleta de dados para serem aplicados junto aos segmentos docente e discente, para aferir o grau do nível de avanço do Curso. O perfil radial avançou para 21 pontos, o que permite subir ao primeiro nível do grau de inovação avançada (Figura 4).

Consensualmente tem-se que o impacto mais positivo da implementação do Pró-Saúde no curso de Odontologia da UEM foi impulsionar o movimento de mudança de forma que, mesmo sem recursos financeiros, ele continue acontecendo. A sustentabilidade das ações deverá permanecer, pois indiscutivel-

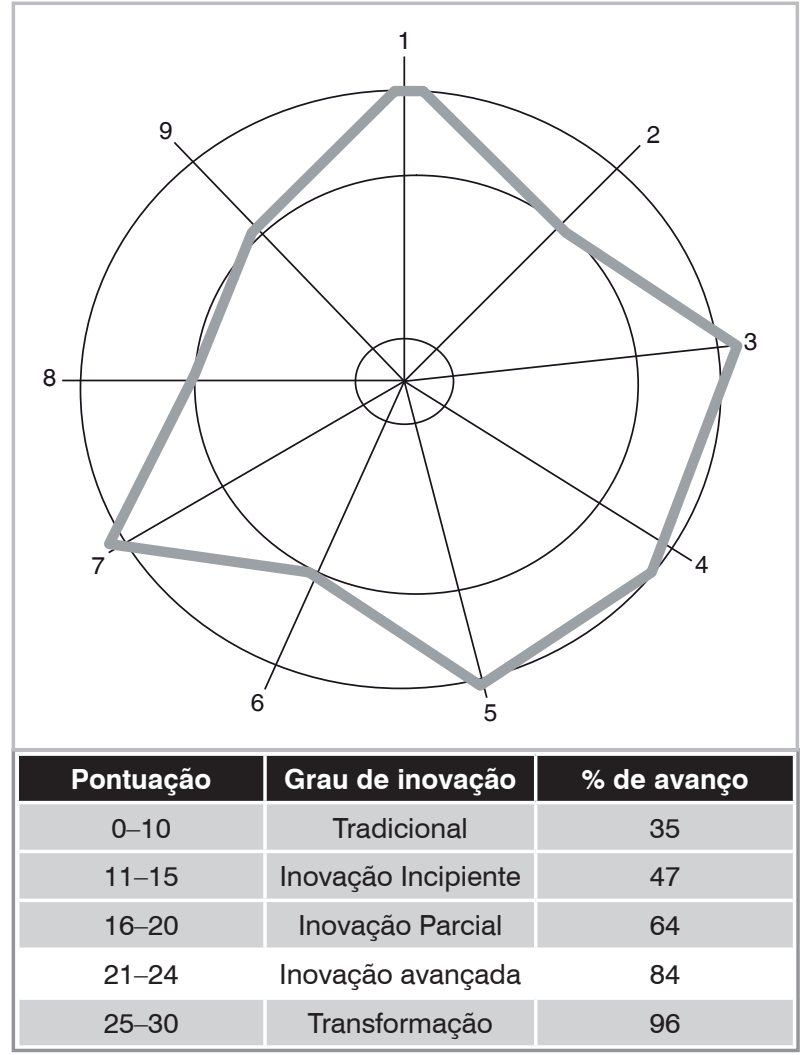

Figura 4 - Perfil radial do grau de inovação em Março/2010.

mente houve uma melhor compreensão do que significa formar recursos humanos para o Sistema Único de Saúde, em consonância com as Diretrizes Curriculares Nacionais.

\section{DISCUSSÃo}

Na busca incessante pela consolidação do Sistema Único de Saúde (SUS) enquanto política de Estado que assegure aos brasileiros as condições de atenção previstas na Constituição Federal, muito foi investido em 20 anos de história. Até pouco tempo atrás, tais investimentos eram fortemente voltados a insumos estratégicos e tecnologia. Não é de longa data que as políticas governamentais voltaram atenção para a formação de recursos humanos em saúde. Apesar da previsão de formação desde a carta constitucional, há aproximadamente 10 anos é que foi iniciada uma ação realmente estruturada para este fim. ${ }^{6}$

A questão da formação de profissionais de saúde envolve diretamente as oportunidades advindas do mercado de trabalho, o perfil profissional e a satisfação das demandas populacionais. Assim, a articulação entre as políticas de educação e de saúde é fundamental para que as transformações sejam possíveis. ${ }^{7} \mathrm{O}$ 
advento das Diretrizes Curriculares Nacionais (DCN's) para Cursos da saúde representa uma destas ações integradas com potencial de gerar frutos para a sociedade. As autoras Senna \& $\mathrm{Lima}^{8}$ ao estudarem os artigos publicados na revista da ABENO sobre as DCN's para o curso de odontologia concluíram que dentre as dificuldades formativas destacam-se a ressignificação do papel da universidade e do ensino de graduação na formação de profissionais de saúde.

A Odontologia no Brasil tem sido sistematicamente criticada por seu caráter excessivamente técnico em detrimento de aspectos fundamentais, como a prevenção, uma relação paciente-profissional mais humanizada e a própria ética do cotidiano. ${ }^{9}$ Neste contexto é que surgiu a proposta de implantação da Clínica Ampliada do Curso de Odontologia da UEM. Embasada na Política Nacional de Humanização e considerando a necessidade de sensibilizar o aluno para outros determinantes do processo saúde-doença é que tal iniciativa se concretizou. Objetiva-se que os frutos dessa formação sejam profissionais cientes da realidade enfrentada pela população e pela saúde pública no Brasil.

Por meio dos convênios firmados entre a Universidade e as Prefeituras Municipais a partir do PróSaúde, foi possível reestabelecer parceria do curso de odontologia da UEM com a rede de serviços do município de Maringá e gerar uma nova parceria com o Município de Marialva. Entendem os docentes do referido curso que a inserção na rede de serviços propicia o trabalho em condições reais de práticas, uma vez que na academia, os alunos trabalham em condições favoráveis para o ensino, o que muitas vezes não representa a realidade do serviço. Corroborando com o exposto, Valente et al., ${ }^{10}$ salientam que as atividades desenvolvidas nas unidades de saúde são fundamentais para a interiorização de práticas do trabalho em saúde, para ensinar, ouvir e criar relações entre serviço-ensino, sendo uma oportunidade ímpar de estimular os discentes a se inserirem no processo de trabalho, de modo a perceberem a atenção à saúde centrada nas necessidades da população. Os autores Gontijo et al., ${ }^{11}$ demonstraram o sucesso de um projeto de saúde bucal coletiva, nos moldes de atuação da estratégia saúde da família para estudantes de uma faculdade privada de Odontologia. Complementarmente destacam Saliba et al., ${ }^{12}$ ao documentar os 50 anos de ações em saúde pública da Faculdade de Odontologia da Universidade Estadual Paulista Júlio de Mesquita Filho, que a integração ensino-serviço, quando presente de forma articulada e planejada, historicamente trouxe benefícios à formação profissional e à comunidade, gerando impacto social.

A inserção da comunidade permanece um desafio em muitas instituições, pois a construção coletiva que a inclua nas discussões e ações é um caminho possível e promissor. $\mathrm{O}$ avanço substantivo na qualidade dos serviços e das ações de saúde só é possível com a participação efetiva da população, pois esta pode apontar problemas e soluções que atendam as suas demandas mais diretamente. Para tanto, o controle social, como espaço político-democrático de representação social sobre os interesses comuns aos cidadãos, deve ser incentivado.

Os pesquisadores Cavalcanti et al. ${ }^{13}$ analisaram a percepção de estudantes de odontologia em relação à atuação no SUS. Concluíram que os estudantes têm uma visão coerente com o perfil profissional esperado para a Odontologia no SUS, segundo as últimas DCN's. Ao enfatizar a humanização e a vivência no SUS, os estudantes consideraram que a formação é direcionada às práticas em saúde pública e desacreditam na eficácia do SUS em atender as necessidades da população. Os autores salientaram ainda existir forte tendência a especialização e ao trabalho articulado entre o setor público e o privado, sendo a autonomia profissional e a lucratividade os principais interesses. De fato, formação de recursos humanos adequados à realidade sócio-epidemiológica do Brasil é o grande desafio para a consolidação do SUS. ${ }^{14}$ Isso é confirmado pelo fato dos estudantes, no lugar de atenderem às necessidades da população, entendem o paciente como instrumento no qual o conhecimento adquirido é simplesmente reproduzido. ${ }^{15}$ Apesar do exposto, há de se considerar que o avanço da Odontologia em Saúde Pública em termos de qualificação de procedimentos, postos de trabalho e empregabilidade cresceu muito nos últimos anos, de modo que o recém formado não possa descartar a possibilidade de atuação neste cenário, principalmente se considerar a, cada dia mais famigerada, competição no mercado de trabalho.

Embasados pelo aporte financeiro e técnico resultante do Pró-Saúde, o curso de Odontologia da UEM participou efetivamente do inédito levantamento epidemiológico em saúde bucal nos municípios de Maringá e Marialva, em parceria com os mesmos. Tal ocorrido propiciou a oportunidade prática de realizar um levantamento nos moldes metodológicos do SB Brasil. O conhecimento do estado de saúde ou doença de uma população é fundamental para se estabelecer um planejamento de atuação em saúde. No Brasil, a Lei 8.080 de 19/09/90, conhecida como 
lei orgânica de saúde, determina (art. $7^{\circ}$, VII) a

"utilização da epidemiologia para o estabelecimento de prioridades, a alocação dos recursos e a orientação programática”. ${ }^{16}$

Aliar a importância do levantamento em diagnosticar saúde bucal à execução do mesmo em âmbito educacional representou uma rica experiência.

Nas iniciativas propostas no Pró-Saúde Odontologia UEM, os envolvidos têm constantemente atribuído atenção às avaliações periódicas das ações, o que tem ocorrido com a participação de todo o corpo docente e auxílio da assessoria pedagógica de formação de recursos humanos. Com vistas à Educação Permanente dos atores, muitas destas avaliações ocorreram no modelo de oficinas e fóruns contando com a participação de representantes do ensino, serviço e comunidade. Segundo Ceccin, ${ }^{17}$ a Educação Permanente em Saúde vem também ao encontro das novas diretrizes curriculares propostas aos cursos de graduação na área da saúde, pois destina-se à transformação do modelo de atenção a saúde, fortalecendo a promoção e prevenção de agravos no Sistema Único de Saúde. Busca também a formação de um profissional crítico, capaz de aprender a aprender, de trabalhar em equipe, de levar em conta a realidade social para prestar uma assistência humana e de qualidade.

De um modo geral, a implantação, adequação e avaliação das ações decorrentes do projeto Pró-Saúde no curso de Odontologia da UEM propiciam constantes reflexões sobre a formação profissional, objetivando a consolidação formativa de um cirurgiãodentista com domínio técnico-científico, conhecedor da realidade social e do Sistema de Saúde Brasileiro, dotado de sensibilidade social e com competências e habilidades para bem atuar no mercado de trabalho.

\section{CONCLUSÕES}

A implantação do projeto Pró-Saúde no Curso de Odontologia da UEM se constituiu em importante marco inspirador de mudanças de pensamentos e práticas, e a implementação deste processo vem ocorrendo gradativamente, sempre visando o fortalecimento da tríade ensino/serviço/comunidade. Criouse uma massa crítica de docentes engajados com o movimento de mudança na formação dos profissionais de saúde e as ações têm sido planejadas e executadas de forma colegiada. Neste processo, persistem alguns desafios, sendo os principais, a integração multiprofissional e a mudança do paradigma curativista para o de promoção de saúde.

\section{ABSTRACT}

Implementing the "Pró-Saúde" program in the Dentistry Course at State University of Maringá

The aim of this study was to report on the implementation of the "Pró-Saúde" Program at the State University of Maringá (UEM), and the activities performed up to 2010. This is a retrospective documentary study that examined technical and financial reports on two letters of agreement for the program. Six teachers were in charge of evaluating all the material and listing the main actions developed. The fundamental actions in this process were the establishment of management and monitoring committees and the hiring of administrative and teaching advisers in the area of human resources. Other key actions included: performance in extramural activities, reintegration of academics in the Maringá municipal health services, establishment of a partnership with the Marialva Department of Health, creation of the "Clínica Ampliada em Odontologia" (Extended Dental Clinic), forums for reporting on the experience obtained and for evaluation, epidemiological surveys and external control of fluoride levels in the Maringá public water supply. It was concluded that the implementation of the "Pró-Saúde" program was a landmark for establishing a critical mass of teachers engaged in the training of health professionals. Remaining challenges are to achieve multidisciplinary integration and make a shift from a curative to a health promotion paradigm.

\section{DESCRIPTORS}

Health manpower. Dentistry. Higher education policy.

\section{REFERÊNCIAS}

1. Frenk J, Chen L, Bhutta ZA, Cohen J, Crisp N, Evans T, et al. Health professionals for a new century: transforming education to strengthen health systems in an interdependent world. Lancet 2010; 376:1923-1958.

2. Lucchese R, Vera I, Pereira WR. As políticas públicas de saúde - SUS - como referência para o processo ensino-aprendizagem do enfermeiro. Rev. Eletr. Enf. [Internet]. 2010; 12(3): 562-566.

3. Brasil. Ministério da Saúde; Ministério da Educação. Programa nacional de reorientação da formação profissional em saúde - PRÓ-SAÚDE: objetivos, implementação e desenvolvimento potencial. Brasília, 2007.

4. Morita MC, Krieger L, Gasparetto A, Carloto EETC, Hihasi MS, Mesas AE et al. Projeto Pró-Saúde Odontologia: relato das ati- 
vidades iniciais em universidades do estado do Paraná. Rev Espaço para a Saúde. 2007; 8(2): 53-7.

5. Hayacibara MF, Terada RSS, Silva MC, Morita MC, Tanaka E, Krieger L. II Fórum dos projetos Pró-Saúde Odontologia das universidades do estado do Paraná - relato de experiência. Rev Espaço para a Saúde. 2009; 11(1): 54-60.

6. Brasil. Ministério da Saúde. Politica de Recursos Humanos para o SUS: balanço e perspectivas. Brasília: Ministério da Saúde, 2003.

7. Araújo ME. Palavras e silêncio na educação superior em odontologia. Ciênc Saúde Coletiva. 2006; 11(1): 179-182.

8. Senna MIB, Lima MLR. Diretrizes curriculares nacionais para o ensino de graduação em odontologia: uma análise dos artigos publicados na revista da ABENO, 2002-2006. 2009; 45(1): 30-36.

9. Almeida AB, Alves MS, Leite ICG. Reflexões sobre os desafios da odontologia no Sistema Único de Saúde. Rev. APS. 2010; 13(1): 126-132.

10. Valente GSC, Gomes HF, Alves FB. Pró-Saúde: Uma nova experiência educativa na formação profissional. Praxis Educa. 2010; 6(9): 227-237.

11. Gontijo LPT, Almeida MCP, Gomide LRS, Barra RP. A saúde bucal coletiva na visão do estudante de odontologia: análise de uma experiência. Ciênc. saúde coletiva 2009; 14(4): 1277-1285.
12. Saliba NA, Saliba O, Moimaz SAS, Garbin CAS, Arcieri RM, Lolli LF. Integração ensino-serviço e impacto social em cinquenta anos de história da saúde pública na Faculdade de Odontologia da Universidade Estadual Paulista Júlio de Mesquita Filho. RGO. 2009; 57 (4): 459-465.

13. Cavalcanti YW, Cartaxo RO Padilha WWN. Educação odontológica e Sistema de Saúde Brasileiro: práticas e percepções de estudantes de graduação. Arq. Odontol. 2010; 46(4): 224-231.

14. Brustolin J, Brustolin J, Toassi RFC, Kuhnen M. Perfil do acadêmico de Odontologia da Universidade do Planalto Catarinense - Lages - SC, Brasil. Rev ABENO. 2006; 6:70-6.

15. Moimaz SAS, Casotti CA, Saliba NA, Garbin CAS. Representação social de acadêmicos de odontologia sobre a área de Odontologia Social. Rev ABENO. 2006; 6:145-149.

16. Pereira AC. Odontologia em saúde coletiva: planejando ações e promovendo saúde. Porto Alegre: Artmed; 2003. Cap. IV.

17. Ceccim RB. Educação permanente em saúde: desafio ambicioso e necessário. Interface Comum Saúde Educ. 2005; 9 (16): 161-168. 